\title{
Systèmes produit service et économies de fonctionnalité : dynamique d'innovation et échelles territoriales
}

\section{Product-service systems and functional service economy: innovation dynamics and territorial scales}

\author{
Céline Merlin-Brogniart ${ }^{1}$ \\ ${ }^{1}$ Univ. Lille, CNRS, UMR 8019 - CLERSE, celine.merlin@univ-lille.fr
}

RÉSUMÉ. Cet article examine les modèles économiques décrits par les différentes approches des économies de fonctionnalité $(E F)$, puis analyse la dynamique de l'innovation que ces modèles génèrent. Nous mobilisons le cadre théorique de l'innovation en termes de caractéristiques afin de rendre compte de la nature et de la dynamique de l'innovation de ces modèles, ainsi que des échelles d'analyse (micro, méso, macro) retraçant les niveaux pertinents d'application de ces modèles. Cet article suggère qu'un grand nombre de développements pratiques des EF existe, en fonction du nombre d'acteurs et de l'échelle spatiale dans laquelle ces modèles s'inscrivent. Certains d'entre eux ne sont pas véritablement en rupture par rapport au modèle de croissance matérielle intensive, d'autres montent en complexité, et requièrent une coordination à un niveau systémique plus large. La dynamique d'innovation, essentiellement de nature servicielle, dépend de la complexité du modèle économique.

ABSTRACT. This paper examines the economic models described by the different approaches of the Functional Service Economy (FSE) and analyzes the innovation dynamics that these models generate. The theoretical framework of innovation in terms of characteristics is mobilized in order to explain the nature and dynamics of innovation of these models, as well as the scales of analysis (micro, meso, macro) that retrace the relevant levels of application of these models. This article suggests that a large number of practical developments of FSEs exists, depending on the number of actors and the spatial scale in which these models are embedded. Some of these models do not provide a real change with the intensive material growth model. Others are becoming more complex and require coordination at a broader systemic level. The dynamics of innovation, essentially of a service-oriented nature, depend on the complexity of the economic model.

MOTS-CLÉS. Innovation, Économie de fonctionnalité, Territoire, Développement durable, Modèle économique. KEYWORDS. innovation, Functional Service Economy, territory, sustainable development, business model.

\section{Introduction}

Le modèle de croissance matérielle intensive, qui a servi de socle à la période de croissance de l'après-guerre, basé sur le volume, ne correspond plus aux attentes socio-économiques actuelles. De nouveaux modèles à visée opérationnelle ont été développés dans les années 1980, en vue de renouer avec la croissance économique, et/ou de répondre aux préoccupations de développement durable. Nous nous intéressons dans cet article à l'un de ces modèles, popularisé en France plutôt sous le nom «d'économie de fonctionnalité » ou «d'économie de la fonctionnalité ». Mais d'autres termes ${ }^{1}$ sont utilisés pour décrire ce modèle qui a pour caractéristique de s'appuyer sur une logique servicielle, privilégiant, pour une même fonctionnalité, la vente d'un service à la vente d'un bien. L'ADEME [ADE 17] qui considère l'EF comme un modèle économique d'avenir, le définit comme

\footnotetext{
${ }^{1}$ L'abréviation «EF » sera employée dans cet article au sens large, autrement dit, pour désigner l'ensemble des approches théoriques de "l'économie de (la) fonctionnalité » et des "systèmes produits-services " (SPS). Certains auteurs utilisent le terme "les économies de fonctionnalité " pour insister sur la pluralité de ces approches. Leur diversité est accentuée dans la mesure où ces approches sont issues de plusieurs disciplines (ingénierie, économie, management).
} 
un modèle qui « consiste à fournir aux entreprises, individus ou territoires, des solutions intégrées de services et de biens reposant sur la vente d'une performance d'usage ou d'un usage et non sur la simple vente de biens. Ces solutions doivent permettre une moindre consommation des ressources naturelles dans une perspective d'économie circulaire, un accroissement du bien-être des personnes et un développement économique ».

Les développements opérationnels de ces modèles sont aussi très variés. Ils dépendent du nombre d'acteurs impliqués et des objectifs recherchés par ces acteurs. Par exemple, certaines entreprises optent pour l'EF à la recherche de nouvelles opportunités de croissance dans l'objectif d'augmenter leur chiffre d'affaires, en remplaçant la vente d'un bien par la vente de l'usage de ce bien sans pour autant envisager un développement durable. D'autres entreprises s'associent à des prestataires de services, pour proposer la vente d'une solution intégrée multi-acteurs, centrée sur l'usage, plutôt que la vente d'un volume de bien, là aussi sans toutefois avoir pour préoccupation principale les avancées sociales ou environnementales. Enfin, certaines entreprises de services, associations ou/et collectivité territoriale coopèrent et adoptent la logique de service en réfléchissant à de nouvelles pratiques prenant en compte des enjeux collectifs, tels que l'amélioration du bien-être des individus ou de l'environnement, même si cette amélioration intervient au détriment de la performance économique. Elle nécessite la création d'un écosystème d'affaires et induit un nouveau mode d'évaluation des performances.

Cet article a pour objectif d'appréhender la diversité des modèles d'EF en proposant une typologie de la dynamique d'innovation que ces modèles engendrent en fonction des échelles envisagées pour le déploiement du système d'offre. Afin d'analyser cette dynamique de l'innovation, nous mobiliserons le cadre théorique de l'innovation en termes de caractéristiques [GAL 94] [GAL 97]. Ce cadre permet de rendre compte de la nature de l'innovation de ces modèles, ainsi que des différentes échelles d'analyse (micro, méso, macro) correspondant aux niveaux d'application des modèles économiques d'EF. Sur le plan méthodologique, nos réflexions proviennent principalement des recueils d'expériences disponibles sur les sites des Clubs d'économie de la fonctionnalité, ainsi que d'études de cas réalisées dans le cadre d'autres travaux [MER 17].

Dans une première section, nous présentons les différentes approches de l'EF. Dans une seconde section, nous identifions les principales caractéristiques de ces modèles économiques. Dans une troisième section, nous présentons la nature de l'innovation que ces modèles économiques génèrent, en relation avec l'échelle territoriale sur laquelle ils s'appliquent.

\section{Les approches de l'économie de la fonctionnalité}

L'EF est un concept non encore stabilisé, qui a été investi par plusieurs approches théoriques. Ces approches se rejoignent quant à l'importance de basculer vers une logique servicielle plutôt que de vendre un bien (ainsi, les droits de propriété attachés aux biens faisant l'objet de la transaction, ou à l'équipement nécessaire pour la transaction, ne sont pas transférés), mais les objectifs recherchés sont appréhendés différemment selon l'approche théorique envisagée. Par ailleurs, ces approches discriminent les cas étudiés en fonction du périmètre de responsabilité choisi par les producteurs.

A l'origine, l'EF est un des axes de réflexion qui est apparu suite au Club de Rome, en lien avec l'économie circulaire et le bouclage du cycle de vie des produits [VAN 14] [BOU 14]. L'expression " économie de fonctionnalité » (service economy) est attribuée à Stahel et Giarini [GIA 86] [GIA 89]. Le concept de l'EF [STA 94a] [STA 97] [STA 06] a été associé dès cette période à deux éléments, la logique de service d'une part, l'environnement de l'autre. Ainsi, substituer la vente d'un service à la vente d'un bien matériel, peut permettre une réduction des consommations de matières 
premières et d'énergie, en créant « une valeur d'usage la plus élevée possible pendant le plus longtemps possible, tout en consommant le moins de ressources matérielles et d'énergie possible ${ }^{2}$ » [STA $06:$ 145]. En effet, l'entreprise, en gardant la propriété du produit, et en assurant la maintenance, augmente la durée de vie de ce produit et assure son recyclage.

Suite à ce premier développement, plusieurs approches théoriques ont émergé, donnant à ce concept un contenu relativement différent. Ces approches sont ici rassemblées en trois grands groupes. Notons que les frontières entre ces approches sont à géométrie variable, même au sein d'un même groupe, car les typologies proposées par les auteurs se chevauchent parfois, ou s'étendent en fonction des dimensions envisagées (prise en compte de la dimension environnementale, ajout de la sphère non marchande, collaborative, offre orientée usage, fonction ou performance $)^{3}$.

\subsection{Les « systèmes produit-service » (SPS) (ou Product-Service Systems-PSS)}

Les travaux pionniers de Giarini et de Stahel [GIA 89] ont donné lieu à une importante production scientifique autour de la désignation anglo-saxonne de «PSS (product-service systems) [HOC 99] qui peut être associée au concept d'EF. Ces conceptions se sont développées notamment dans des laboratoires de recherche installés aux Pays-Bas et en Suède [SID 17]. Face à l'épuisement des modèles productifs de type fordiste s'appuyant sur les gains de productivité et la vente au volume; et en vue d'obtenir de nouvelles opportunités de croissance, ou de se démarquer de la concurrence ; certaines entreprises introduisent une stratégie de servicisation ou « servitization » des biens [WHI 99] [TOF 02], ou de «services autour des produits » [VAN 88] [AND 95] [FUR 97]. Historiquement, la fourniture de services additionnels au produit vendu (facilités de crédit, maintenance, service après-vente...) a été très tôt pratiquée par certaines entreprises. Elle s'insère dans le processus de servicisation de l'industrie et du secteur agricole [GIA 89]. Dans l'approche systèmes produit-service, l'obtention d'une croissance compétitive durable peut s'obtenir aussi bien par l'utilisation d'un (ou plusieurs) biens que de services pour établir la fonction proposée [GOE 99] [MON 02] [BAI 07]. Ces systèmes d'offre peuvent être développés par une société ou en partenariat avec d'autres sociétés, voire impliquer des parties prenantes supplémentaires comme les consommateurs [TUK 06] [TIS 02]. Parmi les SPS, seuls les développements dans lesquels l'entreprise s'engage dans une logique de vente de l'usage plutôt que du bien lui-même peuvent être associés au concept d'EF. Cette évolution implique un changement de l'organisation de l'entreprise. Ainsi, seuls les SPS « orientés usage » ou « orientés résultat», c'est-à-dire n'impliquant plus un transfert de propriété du bien associé à la prestation, constituent une situation d'EF [TUK 04]. Les SPS « orienté usage » vendent l'usage du produit, non le produit lui-même (activité de type location, leasing, mutualisation et partage). Les stratégies relevant des SPS orientés usages sont plus souvent indépendantes de la préservation de l'environnement [BOU 05]. Les SPS « orientés résultat » sont plus élaborés car l'entreprise s'engage sur la vente d'une capacité à produire un résultat (le producteur garantit la satisfaction des besoins du consommateur, quelle que soit la manière de le satisfaire), c'est-à-dire par l'usage d'un bien matériel et/ou d'un service (ex : Facility Management Services), ou la vente d'une performance (ex : économie d'énergie réalisée) [HOC 99]. Comme le constate Stahel [STA 06], ces approches en termes de SPS ne correspondent pas aux travaux pionniers. Elles sont plutôt issues de l'économie industrielle, et l'internalisation par les acteurs économiques des responsabilités et des coûts n'est pas nécessairement une préoccupation de ces travaux.

\footnotetext{
${ }^{2}$ Traduit par Van Niel [VAN 07]

${ }^{3}$ Pour une synthèse complète de l'ensemble de ces approches, voir Serra B. [SER 18], Perspectives durables et territoriales des économies de fonctionnalité légitimes. Une interprétation en termes conventionnalistes. Thèse dirigée par le Pr. BUCLET, Université Grenoble Alpes, 18 juin.
} 


\subsection{L'économie de fonctionnalité}

Cette approche théorique est dans la droite ligne du concept développé par Stahel de «Functional Economy » dans les années 1980, puis porté en France par D. Bourg à la fin des années 1990. Cette approche s'est plutôt adressée, dans ses applications, à de grands groupes industriels. Comme dans le cas des SPS, le passage d'une entreprise industrielle à l'économie de fonctionnalité s'obtient en remplaçant l'appropriation privative du bien vendu par l'accès à son usage [BOU 05] [DUT 06] [VAN 14]. Mais ce changement de stratégie est mis en place dès sa conception, dans une logique d'allongement espéré de la durée de vie des produits manufacturés. Par ailleurs, la relation à l'environnement a évolué. Le concept d' « économie de fonctionnalité » avait au départ pour objectif de « renouveler l'industrie » par la logique servicielle de consommation à l'accès de biens durables [GIA 89], mais ce concept s'identifierait davantage depuis les années 2010 à la logique d' " économie circulaire » [MCD 11] [SID 17]. Cette approche a proposé, depuis le milieu des années 1990, un grand nombre d'études empiriques s'attachant à analyser les différents développements de ce modèle économique depuis le leasing de produits, l'éco-conception de produits ou la contractualisation au résultat [VAN 07] ${ }^{4}$.

L'approche des services éco-efficients (Eco-efficient services) apparue aussi au début des années 1990, peut être associée à l'économie de fonctionnalité, car le registre de la performance lié à l'intégration de service est en lien direct avec la réduction de l'impact environnemental de la consommation [STA 94b] [HOC 99] [MEI 94] [MEI 00] [BAR 03]. En ce sens, elle répond davantage aux objectifs originels de Giarini et Stahel. Mais elle est aussi assimilée à l'approche des SPS dans la mesure où Tukker [TUK 04] englobe ces approches dans sa typologie de SPS. Ainsi, les applications, comme dans le cas des SPS, peuvent correspondre à un cadre contractuel simple (location de courte durée, leasing, ventes fonctionnelles) $)^{5}$, ou prendre la forme d'une « contractualisation au résultat » dans laquelle la responsabilité du prestataire est plus étendue [VAN $14]^{6}$. Une activité de service peut aussi repenser son modèle dans le cadre de l'EF en contractualisation son offre à la performance, si ce n'était pas le cas auparavant. De plus, les notions de SPS et d'économie de fonctionnalité sont parfois confondues car les liens entre les SPS et l'environnement ont été explorés dès le début des années 2000. En 1999, Goedkoop et al. analysent les qualités économique et écologique des SPS et concluent que ces systèmes peuvent alléger la pression environnementale liée à la croissance économique [GOE 99]. A partir de cette période, certains auteurs intègrent l'enjeu environnemental dans leur définition, à l'instar de Mont [MON 04], selon laquelle «Un PSS est un système de produits, services, réseaux d'acteurs et infrastructures qui cherche continuellement à être compétitif et à satisfaire les besoins des consommateurs, et qui a un impact environnemental inférieur à celui des business model traditionnels. » ([MON 04] traduit par Van Niel [VAN $07: 8]$ ).

De nouveaux développements de l'économie de fonctionnalité [VAN 14] [MAI 14] [BUC 14] [MAI 16] ont introduit de nouvelles dimensions, en particulier l'intégration de modèle d'économie non marchande dans leur modèle, ainsi que la notion de territoire. Ces modèles précisent la notion d'éco-conception des modèles économiques d'EF, avec l'éco-conception d'un «système » à

\footnotetext{
${ }^{4}$ Ex : Voir Van Niel [VAN 07], avec par exemple le leasing de photocopieurs par Xerox [FIS 00] ; les études sur le car sharing [BEL 01] [JAC 02] ; le leasing d'appareils électro-ménagers d'Electrolux Euroclean, [MEI 96], l'offre d'un service de gestion et maintenance des pneumatiques destinée aux entreprises de transport de Michelin [BOU 05] ; le Safetainer de Safechem [KRA 95], ou encore les services de contractualisation énergétique [RUD 00].

${ }^{5}$ Ex : Electrolux, qui vend des cycles de lavage

${ }^{6}$ Tels que la performance énergétique des Sociétés de Service en Efficacité Energétique, ou les contrats de confort thermique, de niveau d'éclairage (ex : CLARlight).
} 
l'échelle d'un territoire, ou la co-conception d'une fonction avec une production locale des supports physiques, voire une autoproduction [BUC 14], ou insistent sur les changements de comportements des consommateurs dans les systèmes coopératifs [VAN 14]; ou encore analysent la place attribuée à l'économie de fonctionnalité collaborative dans la typologie des modèles économiques d'EF[MAI 14] [MAI 16].

\subsection{L'économie de la fonctionnalité (et de la coopération) (EFC).}

Ce développement a été proposé essentiellement en France, à partir du début des années 2000, par C. Du Tertre [DUT 06] [DUT 11], le Club de l'Économie de la Fonctionnalité et du Développement Durable, et le cabinet ATEMIS. Ces acteurs ont créé l'Institut Européen de l'Economie de la Fonctionnalité et de la Coopération (IEEFC) ${ }^{7}$. Selon Sidoli [SID 17], l'approche de l'économie de la fonctionnalité et de la coopération est une approche "alternative». Les tenants de ces deux approches ont travaillé ensemble sur différentes études comme le «Chantier 31 » du Grenelle de l'Environnement dédié à l'« économie de fonctionnalité ». Ces approches mêlent toutes deux sciences sociales et politique. L'approche de l'économie de la fonctionnalité (appelée pendant une période «économie de la fonctionnalité et du développement durable» puis «économie de la fonctionnalité et de la coopération ») est toujours adossée à la logique servicielle. Zacklad insiste sur le fait que cette dynamique servicielle soit ancrée dans une acception plus stratégique (recherche de parties prenantes, y compris d'acteurs indirects de l'offre, tels que les collectivités territoriales) que sectorielle de l'approche service. En 2007, cet auteur définissait une offre d'économie de fonctionnalité (appelée plus tard EFC) comme « une offre de produit, de service ou de produitservice, inscrite dans une dynamique servicielle et fournie par un groupement de parties prenantes sur la base de la gestion coopérative d'un bien partagé indivisible résultant des externalités positives associées à la mise en œuvre de cette offre dans la durée » [ZAC $07: 2]$.

L'EFC comporte deux stades de développement. Le premier stade « consiste à passer de la vente de biens ou de services, dissociés les uns des autres, à la contractualisation d'une performance d'usage et/ou d'effets utiles fondée sur leur intégration. ». Ce stade correspond au concept d'EF proposé par Stahel. Le second stade cherche «à élargir le périmètre des activités et des acteurs de telle manière que le saut systémique que cela engage permette à l'écosystème productif de prendre en charge des externalités négatives environnementales et sociales, repérables sur un plan territorial. $^{8}{ }^{\prime}$. Selon Sidoli [SID 17] c'est ce deuxième stade qui caractérise 1'EFC au travers de la notion de solution territorialisée [DUT 11] intégrant biens et services. Les investissements immatériels sont valorisés, tels que les effets positifs obtenus grâce à la formation du personnel, ou encore l'évolution des dispositifs d'évaluation et des dispositifs organisationnels. Dans cette approche, l'objectif est d'engendrer des dynamiques de coopération entre des acteurs hétérogènes, privés ou publics, autour d'une même problématique, appelée "sphère fonctionnelle » au sein d'un système productif [DUT 11] ${ }^{9}$. La coopération entre ces acteurs peut donner lieu à une offre qui répond à des besoins précis et trans-sectoriels d'un territoire (santé, logement, mobilité, éducation...). Enfin, l'EFC s'inscrit dans une perception plus large du développement durable incluant la transformation et le bien-être au travail. D'un point de vue opérationnel, les recommandations effectuées par les tenants de l'EFC s'adressent plutôt aux PME (Petites et

\footnotetext{
${ }^{7}$ Cette association, loi 1901 regroupe des chercheurs, des consultants, des collectivités territoriales, ainsi que plusieurs clubs de la fonctionnalité territoriaux.

${ }^{8}$ https://www.club-economie-fonctionnalite.fr/accueil/d\%C3\%A9finition-de-I-\%C3\%A9conomie-de-la-fonctionnalit\%C3\%A9/

${ }^{9}$ Par exemple Gesnord cherche à faire évoluer le métier d'un centre d'appel téléphonique médical vers la prise en compte des enjeux de santé en combinant la fonction « réception d'appel » avec la prise en compte du planning des médecins d'un territoire (lutte contre les déserts médicaux, bien-être des professionnels, RSE).
} 
Moyennes Entreprises) qui s'inscrivent dans une dynamique territoriale, que ces PME soient issues du secteur manufacturier ou soient déjà une activité de service (cabinet d'avocat par exemple) [SID 17].

Le point suivant présente les principales propositions de création de valeur des économies de fonctionnalité.

\section{L’EF en tant que modèle économique}

La notion de modèle économique rend compte des mécanismes de création de valeur vers lesquels l'entreprise se dirige. Un modèle économique comporte plusieurs composantes : la proposition de valeur composée d'un ensemble de produits et/ou services, la fabrication de la valeur reposant sur une combinaison de ressources et de compétences, la captation et la rémunération de cette valeur et la dimension organisationnelle prenant appui sur un réseau d'acteurs [LEC 06] [DEM 08]. Nous proposons dans cette partie de mettre en relief les éléments sur lesquels porte la création de valeur. Selon les approches théoriques, les propositions de valeur sont plus accentuées vers l'une ou l'autre des dimensions. Cependant, étant donné les multiples croisements existants entre ces approches, il n'est pas possible de définir des éléments discriminants pour chacune des approches précédemment identifiées.

\subsection{Une proposition de valeur fondée sur une logique servicielle}

Le déterminant commun de ces modèles économiques est de reposer sur une offre composée d'un ensemble de produits et/ou services dans lequel la dominante servicielle est essentielle. Pour relever de l'EF, cette logique de servicialisation suppose un changement dans la structure du modèle économique. Autrement dit, pour la quasi-totalité des conceptions d'EF, la création de valeur ajoutée repose sur la modification de l'offre qui substitue la vente d'une fonction d'usage (un service) à la vente du bien lui-même [ERK 04] [BOU 05] [RIF 00] [DUT 11].

Cette amélioration du système productif serait obtenue en sélectionnant les formes (ou processus) de production couplant biens et services les plus rentables, le but étant d'augmenter la marge bénéficiaire de l'entreprise [VAN 07]. Ainsi, l'objectif premier réside dans la maximisation de la valeur du service et la réduction des coûts pour le réaliser. L'objectif commun de ces approches, et en particulier de l'approche SPS, est donc avant tout la recherche d'une performance financière classique de captation de valeur [LUD 10]. L'introduction d'un service ou le passage à une activité de service requiert l'investissement dans des compétences relationnelles ${ }^{10}$, qui peuvent se transformer en compétences stratégiques. La valeur ainsi créée intègre de nouvelles parties prenantes et en particulier le client. La notion de «valeur client élargie » est proposée [LUD 10].

Les nouvelles technologies de l'information et de la communication jouent un rôle essentiel dans ces modèles car elles permettent de collecter les données clients, de proposer la vente fonctionnelle ${ }^{11}$ plutôt que le produit, voire de gérer des problèmes typiques des services comme la gestion de la capacité.

\subsection{Fidélisation du client et lissage de l'activité}

Le passage à un contrat de service suppose une relation de service qui s'inscrit dans la durée, tout comme le partenariat entre les parties prenantes au système d'offre. En matière de fidélisation, la

\footnotetext{
${ }^{10}$ L'échec de certaines entreprises manufacturières à passer à une activité de service est souvent lié au déficit de compétences relationnelles ou partenariales.

${ }^{11}$ Ex : L'entreprise Signature est passée de la vente des panneaux routiers électroniques à l'activité de pourvoyeur d'information (sur l'état de la circulation routière) en investissant dans le champ des TIC [BUC 14].
} 
logique servicielle est totalement différente de la logique industrielle. Alors que la dynamique industrielle s'appuyait sur le conditionnement de masse des consommateurs par l'intermédiaire de la publicité, le passage vers une dynamique servicielle repose sur une logique de proximité et de réseau [ZAC 07]. La nature de la concurrence est de fait modifiée, autrefois basée sur les prix, la concurrence s'établit davantage sur la qualité du service rendu. Par conséquent, le renouvellement de l'achat ne repose plus tant sur les processus marketing et les innovations produits, que sur la confiance, obtenue par la capacité à répondre à des attentes personnalisées, et le développement d'une proximité géographique et culturelle [DUT 07 : 245]. Pour le client, les dépenses sont proportionnées à l'usage, les charges sont donc lissées, les coûts d'accès à l'usage sont variables, les coûts de maintenance sont à la charge du prestataire du service. Pour l'entreprise, le passage à la production de service permet de lisser ses revenus dans le temps, étant donné que les clients souscrivent un contrat à moyen ou long terme avec le prestataire de service. Les revenus de l'entreprise deviennent plus stables et par conséquent, plus prévisibles. Il y a moins d'incertitudes en cas de hausse du prix des matériaux. Les activités de service sont généralement plus rentables, et le profit de l'entreprise est donc accru.

Selon Zacklad [ZAC 07], les stratégies de fidélisation issues de l'économie de fonctionnalité doivent être différenciées des stratégies de fidélisation classiques présentées par Porter [POR 85] qui prennent appui soit sur le volume vendu, soit sur les effets d'intégration, de réseau et d'apprentissage. Les stratégies de fidélisation s'appuyant sur les effets d'intégration, de réseau et d'apprentissage sont plus adaptées aux prestations de service. Elles reposent sur la compatibilité entre les composantes de l'offre (compatibilité du matériel, poids des investissements spécifiques lié à l'installation de ces offres). Cette compatibilité rend le client captif (effets d'intégration et d'apprentissage). Dans le modèle économique de l'économie de fonctionnalité, la fidélisation devient soutenable Zacklad [ZAC 07] ( $c$. tableau 1). Elle prolonge les effets d'intégration et d'apprentissage sans rendre le client captif. Les éléments du bouquet de produits-services deviennent compatibles mais d'autres fonctionnalités sont créées grâce à l'intégration de ces composantes. L'engagement du groupement de parties prenantes porteur de l'offre est alors essentiel, car c'est à partir de leur coordination active qu'apparait la valeur d'usage supplémentaire.

\begin{tabular}{|c|c|c|c|}
\hline & & Segment de marché large & Segment de marché étroit \\
\hline \multicolumn{2}{|c|}{ Coût moins élevé } & $\begin{array}{l}\text { 1. Domination par le coût (stratégie de } \\
\text { volume) }\end{array}$ & $\begin{array}{l}\text { 3.A. Concentration sur les } \\
\text { coûts (idem mais sur une } \\
\text { gamme ou un territoire } \\
\text { restreint) }\end{array}$ \\
\hline \multicolumn{2}{|c|}{ Différenciation } & $\begin{array}{l}\text { 2. Différenciation } \\
\text { (recherche d'avantages perçus comme } \\
\text { uniques par le consommateur) }\end{array}$ & $\begin{array}{l}\text { 3B. Concentration sur la } \\
\text { différenciation (idem mais } \\
\text { sur une gamme ou un } \\
\text { territoire restreint) }\end{array}$ \\
\hline \multirow{3}{*}{ Fidélisation } & Sur le volume & \multicolumn{2}{|c|}{$\begin{array}{l}\text { 4.A. Offres de volume réservées à certains clients sur des gammes } \\
\text { plus ou moins restreintes (par exemple, liées à des abonnements) }\end{array}$} \\
\hline & $\begin{array}{l}\text { Par les effets } \\
\text { d'intégration, de réseau } \\
\text { et d'apprentissage }\end{array}$ & \multicolumn{2}{|c|}{$\begin{array}{l}\text { 4.B. Effet d'intégration lié à la complémentarité et la compatibilité } \\
\text { entre les composantes des offres, effet de réseau pour les systèmes } \\
\text { communiquant, effets liés aux apprentissages. }\end{array}$} \\
\hline & $\begin{array}{l}\text { Soutenable (stratégie de } \\
\quad \text { fonctionnalité) }\end{array}$ & \multicolumn{2}{|c|}{$\begin{array}{l}\text { 4.C. Innovation de fonctionnalité associée à un engagement } \\
\text { contractuel gagnant-gagnant (dynamique servicielle) et à la gestion } \\
\text { coopérative d'un bien partagé indivisible. }\end{array}$} \\
\hline
\end{tabular}

Tableau 1. Présentation des stratégies de fidélisation en complément de celles de Porter [POR 85]

Source : [ZAC $07: 10]$ 


\subsection{Une offre co-produite avec le client}

La dimension servicielle au cœur de ces modèles économiques est mise en évidence dans les conceptions d'économie de fonctionnalité [BOU 05] [BUC 14] [DUT 06] [MOA 06]. Elle s'appuie sur les travaux des économistes français [DEB 94] [REB 97] et les travaux européens en marketing [GRO 98] pour qui la relation de service est primordiale pour caractériser l'activité de service. Ces travaux mettent en évidence un «mode spécifique de création de richesses » dans lequel l'offre est coproduite avec le client. Ainsi, contrairement au modèle fordiste, le client n'est plus « en bout de chaîne mais se trouve au centre du processus de production » [TAN $01: 220]$. L'entreprise met à disposition du client des «fonctionnalités ». La compréhension et gestion des « usages » sont donc au cœur des nouvelles logiques de création de valeur [VAI 10]. La connaissance du client devient nécessaire afin d'adapter au mieux l'offre de solution.

Dans les typologies les plus utilisées [HOC 99] [TUK 04], la coproduction avec le client est plus importante dans le cas d'une prestation orientée résultat qu'une prestation orientée usage. En effet, l'évaluation des résultats et du service rendu étant fondamentale, la recherche d'un résultat augmente la part accordée à la co-production du service, et le mécanisme de rémunération du prestataire peut introduire le partage des économies réalisées, mais aussi des pénalités pour le prestataire lorsque les résultats obtenus sont inférieurs aux objectifs mentionnés dans le contrat [VAN 14]. Ainsi, dans les propositions de valeur orientée usage, l'échelle interrogée pour l'élaboration du modèle économique est généralement l'entreprise (dimension plutôt microéconomique). Elle peut s'étendre à une échelle méso-économique lorsque l'offre implique plusieurs partenaires (valeur orientée résultat). Le réseau de partenaires devient important, la notion d'écosystème d'affaires apparait [MOO 96].

\subsection{Une performance environnementale}

L'élément distinctif des conceptions issues de l'économie de fonctionnalité par rapport aux premiers développements des modèles de SPS est la recherche d'une performance environnementale. En effet, les SPS orientés usage avaient peu de lien avec l'éco-conception. Les liens avec l'éco-conception sont davantage présents dans les SPS orientés résultats. Cependant, il est rare que l'éco-conception soit envisagée de manière systémique. Dans le cas de l'économie de fonctionnalité, la dématérialisation constitue l'essentiel de la performance de la création de valeur. L'objectif de ces offres est de concevoir un processus productif compatible avec un développement durable [MON 02] [MOA 08] [BUC 14]. Pour créer de la valeur d'usage, le producteur va s'orienter vers des modes de conception et de production maximisant la durée de vie opérationnelle de ses produits. Ainsi, les artefacts supports de la prestation gagnent à être rendus adaptables afin de permettre l'intégration d'éventuel progrès technique d'importance sans avoir à changer tout le parc d'équipements associés à la prestation de service [BUC 05a] [BUC 05b] [VAN 07] [ZAC 07]. Dans ces conditions, l'entreprise intègre dès la conception du produit ses divers usages possibles et son recyclage (éco-conception). L'éco-conception peut concerner le support physique associé à la fonction de service, ou bien plus largement le système sans éco-conception du support physique [BUC 14]. Le revenu de l'entreprise devient moins dépendant des ressources matérielles, de l'énergie et des matières premières, et la durabilité des équipements permet d'amortir son coût sur une plus longue période [FRO 10]. L'économie de fonctionnalité introduit donc une nouvelle dimension de création de valeur dans le modèle économique : le développement durable.

Ainsi, ce modèle économique correspond au canal historique de l'EF telle qu'elle a été envisagée suite au club de Rome. La valeur nouvellement créée tient compte de la communauté dans son ensemble puisqu'elle a vocation à réduire les externalités négatives statiques générées par l'activité 
de l'entreprise, ou à limiter les externalités négatives dynamiques ${ }^{12}$. Elle peut aussi, dans une certaine mesure, améliorer les externalités positives, et notamment la valeur sociétale, grâce aux bénéfices sociaux ainsi engendrés. Certains auteurs proposent la notion de valeur triple, ou valeur client étendue [LUD 10]. Selon Lüdeke-Freund [LUD 10], un modèle économique (BM) soutenable est « un BM qui crée un avantage concurrentiel à travers une proposition de valeur supérieure à celle de ses concurrents et qui contribue au développement soutenable de l'entreprise et de la société » La durabilité intègre les attentes de nouvelles parties prenantes [MAI 17]. Dans ces modèles, même si la performance financière reste le principal moteur, la création de valeur recherchée est étendue à la dimension environnementale, qui fait partie du modèle économique. Ce modèle répond aussi, dans une certaine mesure, aux attentes des parties prenantes (clients, fournisseurs, mais aussi communautés locales). Selon Morlat [MOR 16], le déplacement d'une culture du contrat de cession de biens vers un « contrat de service », favorise l'association des fonctions de consommation, de distribution et de production afin de proposer une offre socio-culturelle et écosystémique. La création de valeur de l'entreprise prend de ce fait une dimension d'emblée plutôt méso-économique (prise en compte d'autres acteurs, réduction des externalités négatives liées à l'activité) que microéconomique.

\subsection{Un modèle économique basé sur la coopération}

Le modèle économique associé à l'EFC s'appuie sur une architecture de valeur complexe qui associe une stratégie fonctionnelle, des relations de coopération entre acteurs, avec un ancrage territorial dans le processus productif [MAI 14]. L'entreprise s'interroge, tout comme dans les autres conceptions d'EF, sur l'architecture de valeur à mettre en place (nature de son offre, chaîne de valeur associée, savoir-faire de base à maîtriser, canaux de distribution, compétences à externaliser, coproduction avec le client, etc). De la même manière, le développement durable fait partie de la conception du modèle économique. Ainsi, le découplage croissance/flux de matières, et l'écoconception, sont toujours essentiels dans ce modèle économique, mais l'accent est mis aussi sur les autres dimensions du développement durable et notamment les dimensions sociale et sociétale. Ainsi, la dimension de soutenabilité de ces nouveaux modèles sera perceptible dans les « formes de cohérence qui s'instaurent entre les modes de production et d'appropriation de la valeur ; de mobilisation des ressources humaines et de prise en considération du travail ; de relation marchande et de relation interentreprises (mode de passation de marché avec les fournisseurs, d'une part, et les clients, d'autre part) ; de financement des investissements matériels et immatériels. » [DUT $11: 2$ ]. Selon cet auteur, la valeur considérée n'est donc pas limitée à la valeur dans son expression monétaire car la valeur créée peut comprendre des éléments qualitatifs et non marchands. D'autres approches de l'économie de fonctionnalité, en tenant compte des modèles de consommation collective et des échanges non-marchands, introduisent également ce type de création de valeur [VAN 14] [MAI 14] [BUC 14].

Dans le modèle économique d'EFC, le bien-être au travail, tel que la professionnalisation des salariés, contribue à la création de valeur. Ainsi, l'offre de solution systémique, pour être efficiente, suppose l'instauration d'un nouveau système de management des hommes, et implique une augmentation de la qualification des travailleurs. La main d'œuvre en contact direct avec les clients doit être formée afin de mieux comprendre et repérer les besoins des clients, dans une prise en charge plus globale et de long terme. Les différentes équipes de l'entreprise, (voire des entreprises partenaires), travaillant autrefois de manière séparée (service technique, service commercial), sont amenées à proposer ensemble des solutions personnalisées au client pour prendre en compte

\footnotetext{
${ }^{12}$ Selon Pearce [PEA 76], les externalités statiques ont des effets réversibles sur le bien-être des agents. On peut les internaliser par des accords entre agents économiques contemporains. Les externalités dynamiques entraient des effets persistants sur l'économie (ex : émission de gaz à effet de serre). De ce fait, elles ne peuvent pas être compensés par les mêmes méthodes.
} 
l'ensemble des possibilités de structuration de l'offre. Par ailleurs, la production, pour répondre aux besoins du client, est re-territorialisée [DUT 07]. Ainsi, dans ce modèle économique, la production se fonde sur des principes de coopération plutôt que de concurrence [MAI 14] [GAG 11]. La valeur partenariale est au cour de ces projets. L'entreprise est généralement obligée de s'associer à d'autres entreprises prestataires car l'offre dépasse généralement son domaine traditionnel d'activité. Ainsi, le prestataire initial revoit son organisation (en interne et en externe). Les compétences sont étendues à celles du réseau de partenaires que l'offre implique. Cette collaboration permet une mutualisation des coûts d'investissement et des coûts d'apprentissage, et l'amortissement des investissements consentis dans la durée. L'élargissement de l'offre à de nouvelles parties prenantes est essentielle et nécessite de rendre l'accès au bouquet de produits-services flexible afin d'amortir les coûts (tels que les coûts d'étude, les coûts liés à l'adaptation de l'offre au contexte singulier du bénéficiaire, les mutualisations) [ZAC 07]. Cette flexibilité d'accès, cet engagement délibéré dans la durée participe d'une recherche d'un nouveau mode de développement, qui sort de la logique marchande. Ainsi «L'essentiel de la rentabilité est déterminé par la capacité à contractualiser avec le plus grand nombre d'acteurs possible, le plus grand nombre d'accès au plus grand nombre possible de facettes utiles d'un même phénomène » [MOR 16:177]. Par ailleurs, les effets externes à l'objet social de l'entreprise, qu'ils soient positifs ou négatifs, sont pris en considération dans l'équation. Ces effets résultent de l'activité productive de l'entreprise mais aussi de son insertion territoriale [DUT 11].

\subsection{La construction d'un écosystème territorial et la création d'un patrimoine collectif immatériel}

La prise en charge de solutions systémiques se conçoit à l'échelle d'un territoire. Ainsi, pour être considéré comme EFC, l'intégration d'« effets d'usage » du service doit s'accompagner d'un « déplacement du niveau systémique de la solution » (saut systémique partenarial et spatial) [DUT 11 : 14]. Les solutions proposées portent sur des problématiques comportant des enjeux territoriaux (ex : mobilité, sécurité, santé, éducation, habitat, aménagement du territoire, etc.).

Ces solutions systémiques créent des externalités sociales et environnementales positives pour ce territoire. La logique d'usage des consommateurs est parfois reconsidérée, en relation avec le territoire [MAI 10] [MAI 16]. Ces solutions systémiques, s'appuient sur les ressources locales et font face aux contraintes territoriales. Ainsi, l'articulation des trois axes du développement durable se fait toujours dans un territoire donné, et sous des formes spécifiques à ce territoire [DUT 06].

Ces offres de solutions engendrent le développement d'un « écosystème productif » d'activités, créateur d'externalités sur le territoire. Les effets «médiats » des activités productives dépassent le cadre de l'entreprise. Ils donnent lieu à des bénéfices recherchés par ces partenariats (création d'emploi, réduction de l'impact environnemental), mais peuvent aussi générer des externalités positives.

Le partage et la mise en synergie de ces ressources renforcent le potentiel productif de ces acteurs au niveau d'un territoire ou d'un secteur d'activité. Ils conduisent à la constitution d'un «patrimoine collectif immatériel » [DUT 06] [DUT 07], encore appelé «bien partagé indivisible » [DUT 06]. Lorsque les biens partagés s'étendent aux biens communs d'une collectivité, les acteurs publics (collectivités territoriales, institutions professionnelles représentatives) sont alors parties-prenantes à l'offre globale [ZAC 07].

Ce patrimoine est constitué de ressources stratégiques non mobiles du territoire [DUE 09], encore appelées «ressources spécifiques» [COL 05]. Le partage et cette création de ressources peut concerner des infrastructures, mais dans une logique de service, ce patrimoine comporte essentiellement des investissements immatériels, qui deviennent des ressources pour d'autres acteurs 
du territoire : du capital humain élargi à des problèmes territoriaux ( maturité cognitive », expériences des acteurs); du capital relationnel territorial (ex : relations entreprise-partenaires locaux, des apprentissages collectifs), du « capital innovation » (création de connaissance collective) [DUE 09].

La prise en compte du développement durable complexifie le modèle économique et contraint les acteurs à tenir compte de dimensions méso-économiques (le territoire et les parties prenantes de l'offre) ainsi que de dimensions macro-économiques (l'impact de l'activité sur le plan économique et social). Le passage à une logique de service intégrant les dimensions socio-économiques mais aussi environnementales, demande un changement de référentiel. En effet, la performance des services et son évaluation se modifie du fait du caractère multidimensionnel des enjeux de développement durable [DUT 11]. Pour mettre en œuvre ces offres complexes, le périmètre des externalités à intégrer est à identifier. Les acteurs participant à la création de ces externalités cherchent à être rémunérés en fonction de leur apport au développement durable. Ces nouveaux systèmes multi-acteurs nécessitent la mise en place de nouveaux processus de mesure de la performance. Les dispositifs d'évaluation prendront en compte les ressources immatérielles, les effets médiats des externalités (de moyen et long terme), leur pertinence fonctionnelle et plus généralement le patrimoine immatériel collectif.

L'évolution du modèle économique (et donc de la proposition de valeur) peut avoir un caractère innovant. Le point suivant examine la dynamique d'innovation associée aux économies de fonctionnalité en fonction de la nature de la création de valeur, ainsi que de la dimension (micro, méso macro) qui est impliquée.

\section{La dynamique de l'innovation}

Les modèles économiques découlant de l'EF étant pluriels, il est possible de déterminer différentes dynamiques d'innovation, en fonction de l'échelle envisagée pour le déploiement du système d'offre (l'entreprise, territoire, etc.), ainsi que des éléments de création de valeur qui les distinguent. Cet exercice n'est pas exempt de limites dans la mesure où les auteurs de ces différentes approches peuvent utiliser les mêmes applications empiriques de l'EF pour illustrer les caractéristiques distinctives des modèles économiques qu'ils expliquent. Il s'agit donc ici plutôt d'un exercice analytique.

Boons et Lüdeke-Freund [BOO 13], ainsi que Bocken et al. [BOC 14] ont proposé une typologie des modèles économiques soutenables en fonction du type d'innovation principale réalisée (innovation technologique, innovation sociale et innovation organisationnelle). Dans ces classifications, l'économie de (la) fonctionnalité est considérée comme une innovation sociale, au même titre que le commerce éthique ou la slow fashion, dans la mesure où l'EF permettrait à des consommateurs d'accéder à des produits innovants et coûteux. Ainsi, ces modèles tiennent compte de la communauté (vendre l'usage du bien, se préoccuper du bien être des parties prenantes et encourager la sobriété). Selon ces auteurs, les modèles durables s'appuyant sur l'innovation technologique créent de la valeur grâce à la réduction ou réutilisation des déchets (énergie renouvelable, production bas carbone, écologie industrielle, économie circulaire). Les innovations à dominante organisationnelle se concentrent sur les aspects sociaux de l'entreprise (tels que le modèle d'entrepreneuriat social, les solutions base of the pyramid, ou encore le crowdfunding). Bocken et al. indiquent que l'EF implique en termes de création de valeur, des changements significatifs à l'intérieur de la firme, mais ces auteurs ne différencient pas les types d'EF. Afin de différencier les différents modèles économique d'EF, par les dynamiques d'innovation qu'ils impliquent, nous proposons d'affiner l'analyse des dynamiques d'innovation en utilisant une 
approche intégratrice ${ }^{13}$ : l'approche des services (ou de l'innovation) en termes de caractéristiques. Cette approche permet de partir de la caractéristique commune de ces modèles d'EF à savoir la production d'un complexe de biens-services, pour ensuite décliner les caractéristiques suivant le système d'offre proposé, l'échelle envisagée, ainsi que le niveau de responsabilité choisi par le producteur. Il est aussi possible de préciser si le modèle présenté correspond davantage à l'une des approches de l'EF présentées précédemment, mais comme nous l'avons signalé, les exceptions sont nombreuses. Le premier point présente le modèle théorique, le point suivant expose les dynamiques d'innovation. Enfin, une synthèse des dynamiques d'innovation est proposée en fonction de l'échelle envisagée pour le déploiement du système d'offre.

\subsection{Le modèle théorique}

L'approche des services en termes de caractéristiques est une construction théorique d'inspiration lancastérienne qui fournit une représentation théorique intégratrice de l'innovation dans les biens et les services [GAL 94]. Gallouj et Weinstein [GAL 97] définissent le produit (bien ou service) comme la mise en relation de vecteurs de caractéristiques et de compétences : caractéristiques de service [Y], caractéristiques techniques internes [T] et externes (caractéristiques techniques du destinataire du service) [ $\left.\mathrm{T}^{\prime}\right]$, compétences internes [C] et externes (compétences du destinataire du service) [C'] ( $c f$. Figure 1). L'innovation est envisagée par ce modèle à travers la dynamique de ces caractéristiques, qui peuvent être ajoutées, supprimées, associées, dissociées, améliorées [GAL 02] [GAL 09].

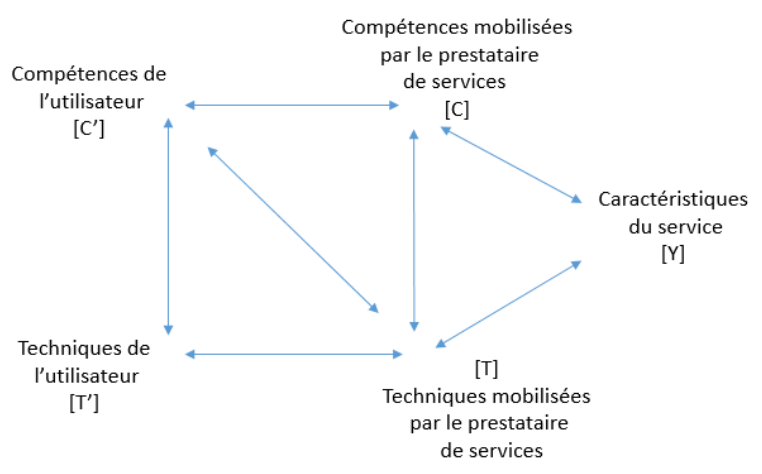

Figure 1. Le produit comme vecteur de caractéristiques et de compétences (characteristics-based approach) Source : [GAL 09] (notre traduction).

Ce modèle rend compte à la fois de services purs basés uniquement sur la prestation de compétences et de connaissances, en coproduction avec le bénéficiaire du service, tout comme des systèmes d'offres de produits-services. Son premier avantage est de pouvoir intégrer les compétences et caractéristiques sociales comme écologiques des services durables. Elle permet notamment de rendre compte de l'amélioration de la soutenabilité d'une activité grâce à l'augmentation de l'intensité en service d'un bien, ce qui diminue la part relative d'activités de traitement de la matière [DJE 09]. Son autre avantage est qu'il peut être utilisé à un niveau micro, méso ou macro-économique. Ainsi, il devient possible de représenter une offre modulant biens et services en intégrant les trois niveaux d'analyse. Ce cadre conceptuel permet ainsi d'affiner les différences de perspectives entre le modèle d'EF.

\footnotetext{
${ }^{13}$ Dans le champ de l'innovation dans les services, l'innovation a d'abord été étudiée par des approches " assimilationnistes " ou "technologistes» (supposant une identité de nature entre l'innovation dans l'industrie et dans les services), puis par des approches "servicielles " (mettant l'accès sur les spécificités de l'innovation dans les services), pour ensuite laisser place à des approches " intégratrices", rendant compte à la fois des spécificités des services comme des dimensions plus matérielles et technologiques des activités de services [GAL 94].
} 


\subsection{Application du cadre théorique de l'innovation en termes de caractéristiques aux différentes échelles analytiques}

\subsubsection{Le passage à une logique servicielle}

Les premiers développements de l'EF ont été entrepris plutôt par des grandes entreprises à la recherche d'opportunité de croissance et sur un segment de marché de l'entreprise. Ainsi, dans ces propositions de valeur, l'échelle interrogée pour l'élaboration du modèle économique est généralement l'entreprise (dimension plutôt microéconomique). La dimension microéconomique (que la Figure 1 illustre) permet d'identifier les modifications liées à l'offre globale de l'entreprise. Nous nous situons au niveau des produits proposés, des changements de configurations productives, et de l'organisation interne de l'entreprise.

Dans le cas des offres orientées usage, le passage à une logique de service conduit à revoir l'organisation interne de l'entreprise, afin de permettre les interactions avec le client, voire une coproduction (par exemple, des départements de l'entreprise peuvent être créés à cet effet). La dynamique de l'innovation s'appuie sur des innovations organisationnelles et servicielles. Graphiquement, il est possible de représenter ce passage d'une logique industrielle à une logique servicielle par l'apparition de vecteurs [C'] et [T'] relatifs aux compétences et techniques mobilisées par le client (comme dans la figure 1 représentée), et d'une amélioration du vecteur [C] par l'investissement en compétences relationnelles. L'introduction d'une dimension servicielle induit la plupart du temps une logique de proximité et de réseau [ZAC 07]. Aussi, lorsque la production de service ne fait pas partie du domaine de base (logique industrielle), l'investissement en connaissances est indispensable pour proposer la nouvelle offre (amélioration des connaissances relationnelles, partage d'expériences, apprentissage). Il constitue les ressources clés des solutions déployées [MER 17]. Cette montée en compétences porte sur la complexité et la pertinence des solutions proposées. La dynamique d'innovation est ainsi servicielle et méthodologique.

Par ailleurs, l'entreprise investit souvent dans les technologies de l'information et de la communication, afin de proposer de nouvelles solutions ou de maîtriser les données clients (et donc d'améliorer la relation de service). Ces améliorations sont retranscrites graphiquement par l'ajout d'éléments relatifs à ces technologies dans le vecteur [T] (adoption d'innovation technologique, technologie informationnelle permettant de partager les données clients par ex.) et du vecteur [C] (investissement en compétences numériques par ex). Dans le cas des offres produits-services «orientées résultat», le niveau envisagé est encore souvent l'entreprise ou l'entreprise et son secteur d'activité. La part accordée à la co-production du service avec le client est plus importante. Elle se traduit par l'augmentation du poids des vecteurs précédemment cités : [C'] [T'] et [C].

\subsubsection{L'introduction de l'éco-conception dans le modèle}

Dans ces modèles, le passage à une logique servicielle peut amener à des bénéfices environnementaux (par la mutualisation des supports physiques entre clients, par l'augmentation de la durabilité des matériaux utilisés par exemple). Cependant, les premières mises en pratique des offres orientées usage n'ont pas mis en avant ces bénéfices comme un résultat recherché. Ces modèles peuvent même être mis en œuvre sans lien avec l'éco-conception, voire augmenter la pression environnementale par l'accroissement de l'utilisation de supports physiques et électroniques. L'accélération de cet usage peut conduire à des effets rebonds ${ }^{14}$ [BUC 05b]. Ainsi, les

\footnotetext{
14 Les économies initialement obtenues par l'utilisation d'une nouvelle technologie (en énergie ou en ressources) sont partiellement ou complètement compensées à la suite d'une augmentation de la consommation du bien intégrant cette nouvelle technologie [SCH 00].
} 
bénéfices environnementaux dégagés au niveau micro-économique, peuvent être plus que compensés par les externalités négatives générées à l'échelle macro-économique.

Dans le cas où l'éco-conception est un résultat recherché de l'offre proposée, les innovations favorisant la réduction des externalités négatives seront identifiables dans le modèle de l'innovation en termes de caractéristiques, par les caractéristiques de service proposées aux clients : vecteur [Y]. Il s'agit de paramètres environnementaux et sociaux tels qu'une offre de formation liée au produit visant à aider le client à en réduire la consommation (ex : réduction de la consommation d'énergie), ou à en prolonger l'usage (ex : lavage du produit), tout comme la prise en charge de la maintenance ou du recyclage du support physique.

L'éco-conception en termes de méthodes et de compétences utilisées pour produire les caractéristiques voulues, sera indiquée dans le vecteur [T] et [C]. L'enrichissement du vecteur [T] peut être obtenu par l'introduction de caractéristiques de durabilité du produit (modularité de l'équipement, facilité de réparation, gestion des déchets). Le vecteur [C] rend compte des compétences de l'entreprise en termes d'éco-conception. Dans le cas où ces préoccupations environnementales intègrent des changements de modes de consommation de la part du client, elles seront également visibles dans les vecteurs [C'] (les compétences des clients mobilisées pour la prestation de service) et le vecteur [T'] (les techniques utilisées par le client de la prestation (ou l'offre globale) de service).

L'éco-conception (du support physique ou du système proposé) est un des objectifs systématiquement mis en évidence par les approches de l'EF et l'EFC, ce qui n'est pas toujours le cas pour les SPS.

\subsubsection{La multiplication des acteurs}

Lorsque les solutions recherchées impliquent plusieurs partenaires prestataires de l'offre de services. L'entreprise va alors développer un écosystème d'affaires. Dans ce cas, l'échelle envisagée sera alors plus étendue (dimension méso-économique). La figure 2 représente une offre multiacteurs (limitée ici au prestataire principal et à un partenaire).

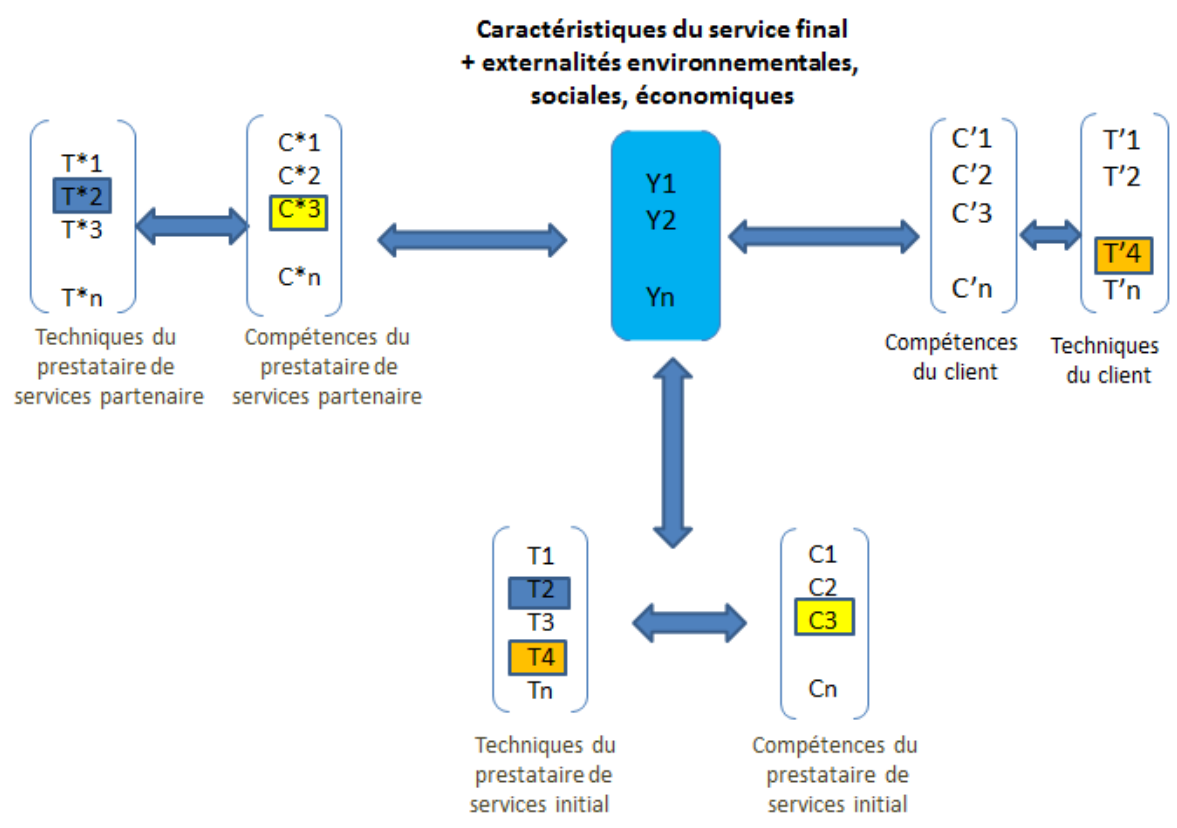

Figure 2. Le produit comme vecteur de caractéristiques et de compétences (Niveau méso-économique). Application au cas d'un système d'offre de fonctionnalité complexe Source : auteur, inspiré de Gallouj, Weinstein, [GAL 97] [WIN 08]. 
En développant un système d'offre de fonctionnalité complexe, l'entreprise externalise certaines parties de la prestation. Elle est, de ce fait, amenée à développer des partenariats avec d'autres prestataires de service ou des industriels. Ces coopérations nécessitent une «synchronisation spatiotemporelle »des acteurs (concernant les horaires, la disponibilité des équipements et plus généralement les ressources nécessaires à la prestation) [ZAC 07].

Graphiquement, il est possible de représenter ces constructions organisationnelles amenant à des solutions systémiques, ainsi que les interactions entre acteurs, par l'utilisation d'autant de systèmes de vecteurs [C] et [T] que de partenaires, avec un unique vecteur [Y] représentant l'offre globale, et les deux vecteurs [C'] [T'] caractérisant le client (que ce soit une offre en B to B ou en B to C). Cette représentation de multiples parties prenantes s'établit à un niveau méso-économique. Cet enrichissement du modèle par l'ajout de partenaires a déjà été entrepris dans les constructions d'innovation de santé afin de rendre compte de l'intervention du secteur public [WIN 08] dans le cas de l'innovation hospitalière [GAL 15], ou encore de l'innovation ferroviaire [DEL 16].

Certaines fonctions, ou sous-ensembles de l'offre, peuvent n'être délivrées que par un des partenaires, ce qui implique des compétences et techniques propres à ce partenaire ; d'autres compétences sont partagées entre parties prenantes (compétences identiques (ex C3 dans la Figure 2), caractéristiques techniques et de process mises en commun (ex T2)). Certaines compétences ou caractéristiques techniques peuvent être partagées avec le client (ex T4). En effet, les clients peuvent avoir un rôle essentiel dans cette structuration de l'offre lorsqu'ils sont amenés à co-concevoir, coproduire et co-évaluer cette offre [DUT 11] [VAI 09].

\subsubsection{La prise en compte du territoire et du patrimoine collectif immatériel}

Lorsque les solutions répondent à des problématiques systémiques et ancrées dans un territoire (cas d'EFC complexes), les constructions organisationnelles sont étendues à d'autres acteurs concernés par la prestation [ZAC 07].

Ces constructions impliquent de nouvelles formes de gouvernance territoriale dans lesquelles les collectivités jouent un rôle d'animateur-facilitateur des coopérations. Ces constructions d'offre ont souvent une approche intersectorielle ou inter-filière. De nouvelles synergies entre acteurs, de nouvelles formes de coopérations publique-privée sont envisagées en vue de créer de la valeur partagée [DUT 07].

Dans ces constructions territoriales, les citoyens peuvent être consultés sur le projet et sur leur habitude de consommation de la fonction considérée. La consultation des citoyens sur certaines fonctions tend à améliorer l'efficacité de la fonction. En effet, dans les enquêtes communément menées, telles que l'enquête déplacement dans le cas de la mobilité, seules les habitudes des personnes enquêtées sont identifiées et non les besoins réels des individus. Autrement dit, les enquêtes révèlent la demande réalisée et non la demande latente [HUG 10]. Cette participation des citoyens a cependant des limites liées à la connaissance des individus sur les offres potentielles et leur usage (par exemple, dans le cas de la mobilité, la connaissance des moyens de déplacements alternatifs et des possibilités de développement de ces offres). 


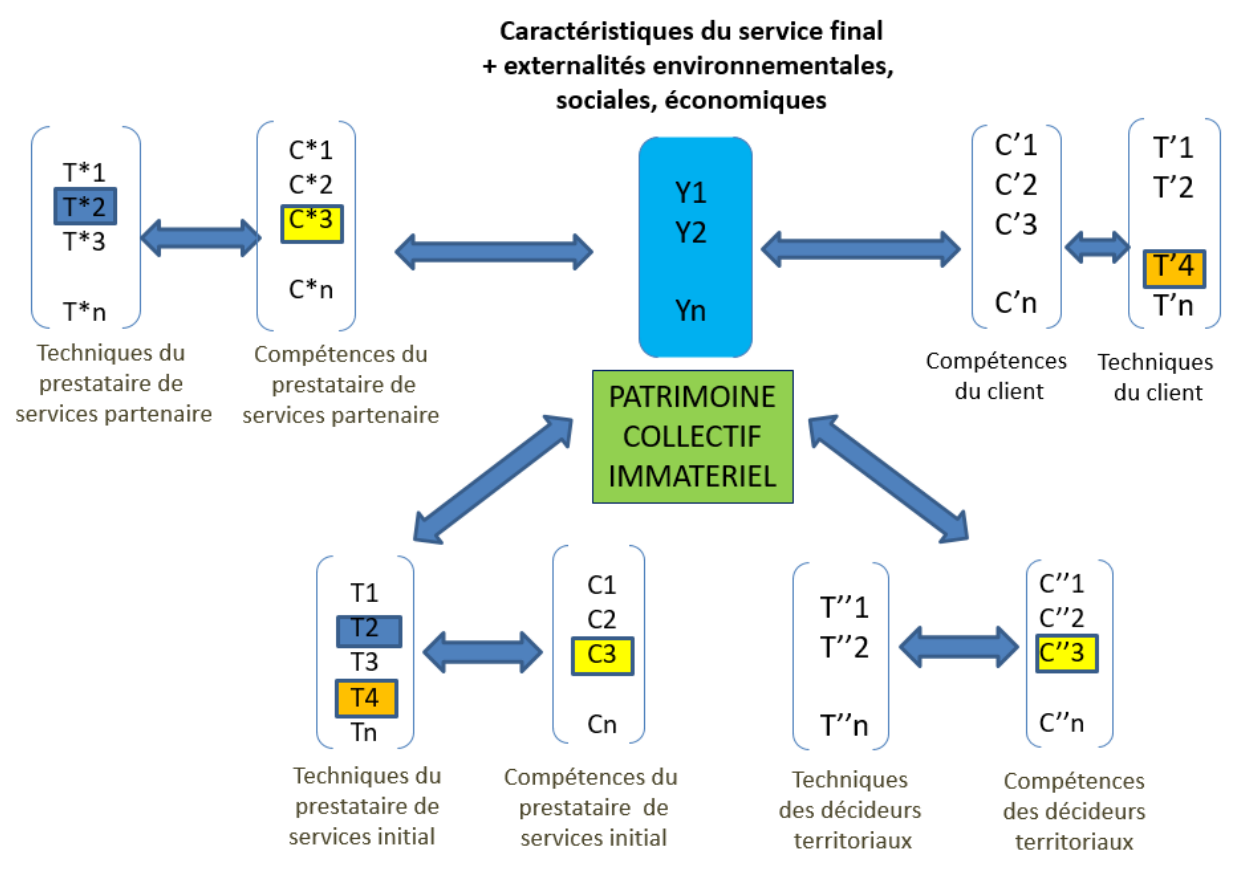

Figure 3. Le produit comme vecteur de caractéristiques et de compétences (Niveau méso-économique). Création d'un patrimoine collectif immatériel

Source : [MER 17], inspiré de Gallouj, Weinstein [GAL 97], Windrum, Garcia-Goñi [WIN 08].

En élargissant le problème traité (ex : en s'intéressant non plus aux moyens de transport mais à la question de la mobilité ; ou non plus au secrétariat médical mais à la question des enjeux de santé, etc.) et en retravaillant la manière d'y répondre (solution systémique multi-prestataires), ces modèles économiques changent l'échelle (micro-méso-macro) considérée. L'élaboration de ces constructions est souvent encadrée par des associations ou des clusters d'économie de (la) fonctionnalité permettant de stimuler la réflexion ou l'émulation de bonnes pratiques (ex : zone de l'Union, Roubaix).

L'objectif de ces constructions d'offre est de générer des bénéfices pour le territoire (création d'emploi, d'activités, cohésion sociale...), ou/et réduire les externalités négatives sur ce territoire [DUT 06] [ZAC 07]. La coopération entre ces acteurs aboutit à la création d'un patrimoine collectif immatériel ( $c f$. Figure 3). Ce patrimoine est représenté dans la figure en tant que résultat de la coopération. On pourrait aussi souligner dans les différents vecteurs, les compétences ou techniques spécifiques associées à ce patrimoine. Le patrimoine collectif immatériel peut être différent en fonction de l'échelle considérée, même si des interactions existent entre les niveaux micro, méso et macro-économiques.

Outre les dynamiques d'innovation déjà présentées dans les paragraphes précédents, une autre dynamique d'innovation, davantage institutionnelle et servicielle, contribue à réduire les effets rebonds attachés à l'innovation technologique. Ceci est rendu possible par la prise en compte plus large de l'implication des effets entre les partenaires sur la zone concernée.

La logique de service conditionne le développement et la pérennité des solutions globales. Elle contribue au déplacement d'échelle de la solution proposée (par la multiplication des parties prenantes, ainsi que le découplage entre la génération de revenus et la consommation de ressources et d'énergie). En effet, les réductions de coûts permis par les améliorations technologiques ne seront effectives que grâce aux prestations de service qui accompagnent la prestation (ex : comme les services de maintenance) [BUC 05a], autrement dit, à la logique servicielle que ce modèle soustend. Par ailleurs, lorsque les externalités sont considérées à un niveau spatial et temporel plus large, 
les solutions choisies incluront non seulement le système de production du prestataire et des parties prenantes (publiques, privées, associatives) à l'offre globale, mais aussi de nouvelles façons de consommer. Nous retrouvons ici l'innovation sociale décrite par Bocken et al. [BOC 14]. La coordination active et volontaire des parties prenantes crée la valeur d'usage supplémentaire, et garantit la pérennité du système [ZAC 07]. Les acteurs mobilisent des savoirs théoriques (scientifiques, techniques, juridiques, commerciaux), développent leurs compétences par la pratique (vecteur $[\mathrm{C}]$ ). Ces savoirs sont obtenus grâce à la variété des acteurs de l'offre globale (ingénieurs, prestataires de services de conseil, collectivités territoriales, associations, etc.). Elle induit une montée en compétences des salariés qui intègrent ce système multi-prestataires.

La dynamique d'innovation institutionnelle est liée aux questions d'évaluation de la création de valeur : lorsque l'EF est inscrite dans une perspective de développement territorial, c'est au niveau de ce territoire que seront recherchés les impacts socio-économiques et environnementaux de l'offre systémique. Les externalités ne peuvent pas être prises en charge (externalités négatives) ou créées (externalités positives) par une firme seule. C'est la coopération entre les parties prenantes qui le permet. Cette coopération implique la présence de dispositifs institutionnels d'évaluation de la performance.

Mesurer et évaluer la création de valeur suppose d'identifier le périmètre des externalités à prendre en compte, le recensement des ressources matérielles/immatérielles et des effets médiats/immédiats à intégrer dans les critères d'évaluation. Un accord sur les acteurs parties prenantes du territoire, qui prendront part à l'élaboration de ces critères (sociale, environnementale, économique) doit être établi, tout comme sur les acteurs qui participeront à l'évaluation des performances a posteriori. Ce dialogue social territorialisé n'est pas toujours facile à obtenir car la solution proposée doit faire consensus, alors que les acteurs ont des priorités, des expertises et des intérêts divergents.

D'un point de vue analytique, la dynamique institutionnelle s'exerce à un niveau macroéconomique. Si le modèle de l'innovation en termes de caractéristiques ne permet pas directement de rendre compte de ces nouveaux systèmes d'évaluation socialement construits, il est possible retranscrire la contribution de chaque acteur au moyen d'un modèle d'évaluation à la fois multi-acteurs et multi-critères ( $c f$. par ex. [HYY 14]).

\subsection{Les dynamiques d'innovation d'EF : une synthèse}

Le tableau 2 récapitule les principales dynamiques d'innovation que l'on peut identifier dans les modèles d'EF en fonction de l'échelle considérée. Ce tableau précise quelques types d'applications de chacune des dynamiques d'innovation de ces modèles. Selon le degré de complexité des projets mis en œuvre, tous les types d'innovations présentés ne seront pas forcément mobilisés.

La dynamique d'innovation servicielle est toujours présente dans les modèles d'EF puisqu'elle forme la base de ces modèles, mais son application est plus ou moins complexe et intègre plus ou moins d'acteurs selon les cas. Cette dynamique rend compte d'un changement de stratégie de l'entreprise vers une logique de service. Elle peut constituer une transformation importante si l'entreprise appartient au secteur manufacturier et introduit des prestations de service de manière conséquente. Elle implique la mise en place de compétences relationnelles de technique de fidélisation qui sont différentes de la logique de volume précédemment utilisée.

La dynamique d'innovation technologique comporte les technologies informationnelles nécessaires à la mise en réseau et à la constitution de bases de données clients/usagers, ainsi que les différents niveaux/échelles attribués à l'éco-conception. L'éco-conception peut se limiter à quelques bonnes pratiques sans effet important sur l'environnement ou être élaborée à une échelle plus large 
dans une perspective d'économie circulaire. La dynamique d'innovation organisationnelle rend compte de la complexité de l'offre et des changements qu'elle implique pour l'entreprise et ses partenaires.

La dynamique d'innovation institutionnelle est particulièrement innovante lorsqu'elle est mise en place dans un processus macro-économique. Elle n'est alors pas le fait des seuls acteurs parties prenantes à ces projets. Elle peut être initiée par ces acteurs mais nécessite une adhésion plus large d'acteurs privés et publics pour qu'une gouvernance traitant des questions d'évaluation et de performance puisse s'instaurer.

\begin{tabular}{|c|c|c|c|}
\hline $\begin{array}{l}\text { Echelle / } \\
\text { Nature de l'innovation }\end{array}$ & $\begin{array}{c}\text { Echelle } \\
\text { microéconomique } \\
\text { (entreprise) }\end{array}$ & $\begin{array}{c}\text { Echelle } \\
\text { méso-économique } \\
\text { (réseau partenarial) }\end{array}$ & $\begin{array}{c}\text { Echelle multiple /Méso- } \\
\text { économique sur un } \\
\text { territoire, voire } \\
\text { macroéconomique }\end{array}$ \\
\hline \multirow[t]{2}{*}{$\begin{array}{c}\text { Servicielle } \\
\text { + Servicisation Passage } \\
\text { de la vente d'un produit } \\
\text { à la vente de l'usage du } \\
\text { produit }\end{array}$} & $\begin{array}{l}\text { Apparition d'un vecteur } \\
\text { [C'] et [T'] du client } \\
\text { Compétences } \\
\text { relationnelles } \\
\text { Professionnalisation } \\
\text { des salariés. }\end{array}$ & $\begin{array}{l}\text { Structuration d'une offre } \\
\text { globale basée sur le(s) } \\
\text { service(s) rendu(s). } \\
\text { Création d'activités } \\
\text { annexes (formation, } \\
\text { conseil). }\end{array}$ & $\begin{array}{l}\text { Structuration d'une offre } \\
\text { globale basée sur le(s) } \\
\text { service(s) rendu(s) et le } \\
\text { territoire. } \\
\text { Apprentissage collectif } \\
\text { territorial, Accumulation des } \\
\text { connaissances territoriales }\end{array}$ \\
\hline & $\begin{array}{l}\text { Utilisation des TIC en } \\
\text { interne }\end{array}$ & $\begin{array}{c}\text { Utilisation des TIC } \\
\text { partagés }\end{array}$ & Utilisation des TIC partagés \\
\hline $\begin{array}{l}\text { Technologique/ } \\
\text { Méthodologique }\end{array}$ & $\begin{array}{l}\text { Si eco-conception, plutôt } \\
\text { limitée à l'échelle de } \\
\text { l'entreprise } \\
\text { Eco-conception }\end{array}$ & $\begin{array}{l}\text { Eco-conception, process } \\
\text { re-engineering } \\
\text { Eco-conception sur les } \\
\text { supports }(\text { vecteur } \mathrm{T})\end{array}$ & $\begin{array}{l}\text { Objectif d'éco-conception } \\
\text { systémique (sur l'ensemble } \\
\text { des vecteurs) et partenarial }\end{array}$ \\
\hline Eco-conception & $\begin{array}{l}\text { sur le support (vecteur } \\
\text { T) parfois dans la } \\
\text { solution proposée } \\
\text { (vecteur Y) }\end{array}$ & $\begin{array}{l}\text { Eco-conception sur le } \\
\text { système (et pas } \\
\text { nécessairement du } \\
\text { support). } \\
\text { Réflexion sur l'ensemble } \\
\text { du cycle de vie du produit }\end{array}$ & \\
\hline Organisationnelle & $\begin{array}{c}\text { Evolution de } \\
\text { l'organisation en interne } \\
\text { Mutualisation des } \\
\text { usages. }\end{array}$ & $\begin{array}{c}\text { Changement } \\
\text { d'organisation incluant } \\
\text { des acteurs externes } \\
\text { Mutualisation }\end{array}$ & $\begin{array}{l}\text { Co-conception d'une } \\
\text { fonction à plusieurs } \\
\text { parties prenantes, acteurs } \\
\text { externes et territoriaux }\end{array}$ \\
\hline Institutionnelle & $\begin{array}{c}\text { Institutionnalisation } \\
\text { Par ex. via un Rapport } \\
\text { de développement } \\
\text { durable }\end{array}$ & $\begin{array}{l}\text { Recherche de nouveaux } \\
\text { critères d'évaluation de la } \\
\text { performance-réflexion sur } \\
\text { la contribution- } \\
\text { rémunération des } \\
\text { différents acteurs }\end{array}$ & $\begin{array}{l}\text { Recherche de nouveaux } \\
\text { critères d'évaluation de la } \\
\text { performance y compris à } \\
\text { l'échelle d'un territoire- } \\
\text { institutionnalisation au } \\
\text { niveau des collectivités } \\
\text { territoriales. }\end{array}$ \\
\hline Exemples & $\begin{array}{l}\text { Location de vêtement } \\
\text { de matériel par un seul } \\
\text { prestataire }\end{array}$ & $\begin{array}{l}\text { Gesnord (centre d'appel } \\
\text { téléphonique médical, } \\
\text { enjeux de santé) }\end{array}$ & Zone de l’Union (Roubaix) \\
\hline
\end{tabular}

Tableau 2. Nature de l'innovation et échelle du système d'offre Source : auteur

La nature des innovations et leur dynamique sont considérées isolément dans le tableau mais sont en réalité liées les unes aux autres, et cumulatives (ex. les TIC rendent possibles certaines 
techniques de fidélisation), les innovations organisationnelle et servicielle sont généralement associées (mises en place de nouveaux départements dans l'entreprise pour générer les services).

Les dynamiques d'innovation sont déclinées selon trois échelles (en colonne). Des changements essentiellement au niveau microéconomique (changements technologiques, organisationnels, capacité d'innovation de l'entreprise, interne à l'entreprise) ; des changements à l'échelle mésoéconomique (performance des services, changements organisationnels, coopération); et des changements macroéconomiques (changements structurels et institutionnels, participation de nombreux opérateurs et régulateurs).

\section{Conclusion}

Dans cet article, nous avons proposé une synthèse des approches de l'EF, en identifiant, les caractéristiques des modèles économiques ainsi que les principales dynamiques d'innovation que ces modèles empruntent. Plusieurs résultats ressortent de cette étude.

Les modèles économiques de l'EF, tels qu'ils se sont construits, n'ont pas eu les mêmes objectifs (dégager des opportunités de croissance par la logique servicielle, engager une entreprise ou un grand groupe dans une croissance soutenable, créer des externalités positives pour un territoire...) ; ni les mêmes cibles (les grands groupes manufacturiers, les PME et leur territoire). Selon ces perspectives, les échelles d'analyse envisagées sont différentes (micro-méso-macro), ainsi que le nombre et la variété des acteurs impliqués.

La dynamique d'innovation sur laquelle ces modèles reposent est avant tout de nature servicielle : le passage de la vente d'un produit à la vente de l'usage de ce produit. Ainsi, la logique servicielle permet de modifier la captation de la valeur et la forme du revenu (lissage du revenu, fidélisation du client). Les effets obtenus dépendront de l'échelle d'analyse envisagée. En particulier, la réduction des externalités négatives dépendra du saut systémique partenarial et spatial effectué par l'entreprise.

Les modèles économiques d'EF sont souvent présentés comme une réponse aux enjeux du développement durable. Or, dans les premiers développements d'EF, les conséquences positives pour l'environnement liées à la dématérialisation n'étaient pas considérées comme un objectif stratégique [ZAC 07]. De nos jours, les bénéfices environnementaux, sociaux et économiques (moindre pollution, moindre consommation de matières et d'énergie création d'emplois locaux, gestion des ressources locales) sont plus systématiquement annoncés par les entreprises même s'ils ne sont pas l'objectif prioritaire de ces conversions à l'EF. D'ailleurs, le passage à l'EF est souvent envisagé par les entreprises lorsque leur situation financière devient difficile et que les autres solutions (restant dans l'esprit de la vente au volume) ont été étudiées. Par ailleurs, de nombreux auteurs expliquent la viabilité environnementale de ces modèles économiques en insistant sur le rôle des innovations technologiques et du progrès technique dans la réduction de l'utilisation des matières premières et de l'énergie (éco-conception, économie circulaire). Cependant, ces innovations technologiques ne sont pas suffisantes pour garantir la soutenabilité de ces modèles. Elles peuvent même conduire à des dérives d'un point de vue environnemental [DIE 12] [DUT 11].

D'autres formes d'innovation non technologiques jouent un rôle essentiel dans le développement de ces nouveaux modèles et l'obtention de bénéfices environnementaux, notamment l'innovation organisationnelle. Les innovations organisationnelles sont généralement indispensables à la mise en pratique de l'EF. Elles accompagnent les innovations technologiques. Leur absence peut expliquer certains échecs de passage à l'EF. Outre les innovations organisationnelles qui accompagnent les innovations technologiques, la bonne coopération entre les acteurs, et la gouvernance des modèles 
économiques d'EF complexes, sont garantes de la pérennité des projets. La dynamique d'innovation organisationnelle est aussi plus ou moins complexe en fonction de la constellation d'acteurs concernés par l'offre globale.

Les applications des modèles de fonctionnalité à une échelle méso-économique proposent d'avoir une vue d'ensemble des externalités négatives, ainsi que des bénéfices émis sur un territoire, et de les traiter de manière systémique. Dans ces projets, l'innovation est aussi de nature institutionnelle, puisque la création d'un "patrimoine collectif immatériel» et sa pérennisation dépendra de la capacité des acteurs à identifier les externalités générées et les évaluer (afin de préciser la contribution et donc la rémunération de chaque acteur à la création d'externalités) [ZAC 07]. Un dialogue social territorialisé peut s'engager afin de modifier en profondeur les repères institutionnels et organisationnels, en déterminant les performances économiques, sociales et environnementales propres au territoire qui seront privilégiées. Ces modes de coopération et les nouveaux modes de consommation et de production qui en découlent, constituent des innovations sociales.

Le développement de ces modèles pose un certain nombre de difficultés. D'une part, ces logiques de résolution de problème, de type coopératif, sont coûteuses (coût de création, coût d'adaptation). L'amortissement de ces coûts ne s'obtient que par la durabilité de l'engagement des relations entre les réalisateurs de l'offre, ainsi que par une mutualisation de ces coûts entre les parties prenantes à l'offre (réalisateurs mais aussi destinataires) [ZAC 07]. Ensuite les difficultés dépendent de l'échelle envisagée. Ainsi, les modèles qui rencontrent le plus de difficultés à être mis en place, puis à se pérenniser, sont ceux impliquant un grand nombre d'acteurs à l'échelle du territoire (modèle économique d'EFC complexe). Les acteurs manquent d'incitations, puisque l'objectif n'est pas uniquement économique mais aussi sociétal et environnemental, et implique la mise en œuvre d'une nouvelle méthode d'évaluation de la performance multicritères (dynamique d'innovation institutionnelle). Cependant, les politiques d'aménagement des territoires valorisent de plus en plus ce mode de développement. Ainsi, cette dynamique institutionnelle est en construction, à la recherche de formes de cohérence [DUT 11] permettant de développer ces coopérations, de créer ces externalités positives sur le territoire, puis de les réguler [MER 17].

\section{Références}

[ADE 17] ADEME, L’économie de la fonctionnalité : de quoi parle-t-on?, Claire Pinet, mai 2017.

[AND 95] ANDERson J. C., NARUS J. A., « Capturing the value of supplementary services », Harvard Business Review, 73, n³, p. 75-83, 1995.

[BAi 07] Baines, T.S., Lightfoot, H., Steve, E., Neely, A., Greenough, R., Peppard, J., Roy, R., SheHAB, E., Braganza, A., Tiwari, A., Alcock, J.R., Angus, J.P., Bast, M., Cousens, A., Irving, P., Johnson, M., Kinsgston, J., Lockett, H., Martinez, V., Michele, P., Tranfield, D., Walton, I.M., Wilson, H. (2007), State of the art in product service-systems, Journal of Engineering Manufacture, 221, n¹0, p.1543-1552, 2007.

[BAR 03] BARTOLOMEO M., DAL MOSO D., DEJONG P.R., ZARING O., Eco-efficient producer services - what are they, how do they benefit customers and the environment and how likely are they to develop and be extensively utilised ?, Journal of Cleaner Production, $11 \mathrm{n}^{\circ} 8$, p. 829-837, 2003.

[BEL 01] BELZ F., Mobility Car Sharing - Successful Marketing of Eco-Efficient Services, St-Gallen: University of St-Gallen, 2001.

[BOC 14] BOCKEN N-M-P, SHORT S-W, RANA P, EvAnS S, « A literature and practice review to develop sustainable business model archetypes », Journal of Cleaner Production, n65, p. 42-56, 2014.

[BOO 13] BOONS F., LÜDEKE-FREUND F., « Business models for sustainable innovation: state-of-the-art and steps towards a research agenda », Journal of Cleaner Production n45, p. 9-19, 2013.

[BOU 05] BOURG D., BuCLET N., «L'économie de fonctionnalité : changer la consommation dans le sens du développement durable », Futurible, n³13, p. 27-37, 2005. 
[BOU 14] Boutillier S., LAPERCHE B., PICARD F., «Le développement des systèmes produits-services dans les entreprises : une étape vers l'économie de la fonctionnalité ? », Economies et Sociétés, Série EGS, 4 n ${ }^{\circ} 15$, p. 551 578, 2014.

[BUC 05a] BUCLET N., «Concevoir une nouvelle relation à la consommation : l'économie de fonctionnalité », Annales des Mines, juillet, p. 57-66, 2005a.

[BUC 05b] BUCLET N., «Vendre l'usage d'un bien plutôt que le bien lui-même : Une piste pour concilier meilleure prise en compte de l'environnement et rentabilité des entreprises », dans XVth International RESER Conference: Growth, Employment and Location of Services: New Trends in a Global World, Grenade (Espagne), 22-24 Septembre 2005b.

[BUC 14] BUCLET N., "L'économie de fonctionnalité entre éco-conception et territoire : une typologie », Développement durable et territoires [En ligne], 5, $\mathrm{n}^{\circ} 1,2014$.

[COL 05] Colletis G., PeCQUeur B., « Révélation de ressources spécifiques et coordination Située », Economie et Institutions, (6-7), 51-74, 2005.

[DEB 94] DE BANDT J., GADREY J. (Eds.), Relations de service, marchés de services, CNRS éditions, coll. Recherche et entreprise, 1994.

[DEL 16] DelePlace, M. (2016), "Grande vitesse ferroviaire et développement local dans des contextes institutionnels différents. Pour une spatialisation des analyses de l'innovation de service» dans Forum de l'Innovation, 20 ans d'Innovation, Paris, 9-11 juin 2016.

[DEM 08] DEMIL B., LECOCQ X., «(Re)penser le développement des organisations. Les apports du modèle économique », Revue Française de Gestion, n¹81, p. 113-122, 2008.

[DIE 12] DIEMER, A., « La technologie au cœur du développement durable: mythe ou réalité? », Innovations, $\mathrm{n}^{\circ} 37, \mathrm{p}$. 73-94, 2012.

[DJE 09] DJellal F., Gallouj F., «Innovation dans les services et entrepreneuriat : au-delà des conceptions industrialistes et technologistes du développement durable », Innovations, 1, n²9, p. 59-86, 2009.

[DUE 09] DUEZ P., « Management territorial des risques et prospective territoriale », Marché et Organisations, $\mathrm{n}^{\circ} 9, \mathrm{p}$. 141-169, 2009.

[DUT 06] DU TERTRE C., « Ouvrir le champ de l'évaluation de la performance au registre des externalités », dans E. HEURGON (coordinatrice), Le développement durable c'est enfin du bonheur, Editions de l'Aube, collection essai, p. 104-120, 2006.

[DUT 07] DU TERTRE C., «Économie de la fonctionnalité, développement durable et innovations institutionnelles », dans. E. HEURGON, J. LANDRIEU (dir.) L'Économie des services pour un développement durable. Prospective, essais et recherche, L'Harmattan, Paris, p. 241-255, 2007.

[DUT 11] DU TERTRE C., "Modèles économiques d'entreprise, dynamique macroéconomique et développement durable », dans Gaglio G., Lauriol J. et Tertre C. du, L'économie de la fonctionnalité : une voie nouvelle vers un développement durable?, Editions Octares, Toulouse, 2011.

[ERK 04] ERKMAN S., Vers une écologie industrielle : comment mettre en pratique le développement durable dans une société hyper-industrielle (2nd edition), Paris, Editions Charles Léopold Mayer, 2004.

[FIS 00] FishbeIn B., MCGARRY L. S., DiLlon P. S., Leasing: A Step Toward Producer Responsibility, INFORM, Duke University, Nicholas School of the Environment, Tufts University, The Gordon Institute. 75 p, 2000.

[FUR 97] FURRER O., «Le rôle stratégique des «services autour des produits » », Revue Française de Gestion, marsavril-mai, p. 98-107, 1997.

[FRO 10] FROMANT E., «Un facteur moderne d'abaissement des coûts: l'économie de fonctionnalité », Environnement et Technique, Stratégie et Management, Vertitude Magazine, n²97, juin, 2010.

[GAG 11] Gaglio G., LAURiol J., DU TERTRE C., «L'économie de la fonctionnalité : une voie nouvelle vers un développement durable? », Octares, 2011.

[GAL 94] GaLlouj F., Economie de l'innovation dans les services, L'Harmattan, Logiques Economiques, 1994.

[GAL 02] Gallouj F., «Innovation in services and the attendant myths », Journal of Socio-economics, 31, p. 137$154,2002$. 
[Gal 15] Gallouj F., Merlin-Brogniart C., Moursli-Provost A. - C., « Les partenariats public-privé et leur place dans l'innovation hospitalière : quels enseignements pour le management hospitalier ? », Innovations, $\mathrm{n}^{\circ} 46$, p. 161- 195, 2015.

[GAL 09] Gallouj F., Savona M., "Innovation in Services: A Review of the Debate and a Research Agenda, Journal of Evolutionary Economics », 19, n², p. 149-172, 2009.

[GAL 97] GALlOUJ F., Weinstein O., «Innovation in services », Research Policy, 26, n²4-5, p. 537-556, 1997.

[GIA 86] Giarini O., Stahel W. R., « The hidden wealth », Science and Public Policy, 13, nº4, p. 83-102, 1986.

[GIA 89] Giarini O., Stahel W. R., The Limits to Certainty: Facing Risks in the New Service Economy, Dordrecht, Kluwer Academic Publishers, 1989.

[GOE 99] Goedkoop M. J., Van Halen C. J. G., Te Riele H. R. M., Rommens P. J. M. (1999), Product Service Systems, Ecological and Economic Basis: Pricewaterhouse Coopers N.V. / Pi!MC, Storrm C.S., Pre consultants.

[GRO 98] GRONROOS C., «Marketing services: the case of a missing product », Journal of Business and Industrial Marketing, 13, p. 322-338, 1998.

[HOC 99] HOCKERTS K., « Eco-efficient Service Innovation: Increasing Business-Ecological Efficiency of Products and Services », dans M. Charter (Ed.), Greener Marketing: A Global Perspective on Greener Marketing Practice, Sheffield, Greenleaf publishing, p. 95-198, 1999.

[HUG 10] HUGUENIN-RICHARD F., «Mobilité urbaine : de l'automobilisme à l'éco-mobilité. Un long chemin... », dans V. Moriniaux, Mobilités, Armand Colin, p 109-137, 2010.

[HYY 14] HyYTINEN K., GALlouj F., TOIVONEN M., "A Multi-criteria and Multi-actor Perspective for the Evaluation of Sustainability Services", 5th International Conference on Applied Human Factors and Ergonomics AHFE 2014, Jul 2014, Krakow, Poland, halshs-01133963, 2014.

[JAC 02] JACOBSSON U., Towards more sustainable mobility: Exploring success factors for car sharing in Europe. M.Sc. thesis, IIIEE, Lund University, Lund. 100 pp, 2002.

[KRA 95] KRANENDONK S., Rent-a-Chemical as a Strategy for Resource Reduction, Wuppertal Institute for Climate, Environment, and Energy, 1995.

[LEC 06] LeCOCQ X., Demil B., WARNIER V., «Le business model, un outil d'analyse stratégique », L'Expansion Management Review, ${ }^{\circ}$ 4, 96-109, 2006.

[LUD 10] LÜDEKE-FREUND F (2010), « Towards a conceptual framework of business models for sustainability », ERSCP-EMU Conference, Delft, The Netherlands, p. 1-28.

[MAI 14] MAILLEFERT M., ROBERT I., «Écologie industrielle, économie de la fonctionnalité, entreprises et territoires : vers de nouveaux modèles productifs et organisationnels ? », Développement Durable et Territoires, 5, n ${ }^{\circ} 1, \mathrm{p} .1-6$, 2014.

[MAI 17] MAILlEFERT M., ROBERT I., « Nouveaux modèles économiques et création de valeur territoriale autour de l'économie circulaire, de l'économie de la fonctionnalité et de l'écologie industrielle », Revue d'Économie Régionale \& Urbaine, n5, p. 905-934, 2017.

[MAI 10] MAILlEFERT M., SCHALChLI, P., « Pré-requis pour la construction d'une méthodologie pour l'implantation d'une démarche d'écologie industrielle à l'échelle d'un espace territorial », dans M. Maillefert, O. Petit, O., S. Rousseau (coordinateurs), 2010, Ressources, patrimoine, territoires et développement durable, Bruxelles, Peter Lang, p. 45-68, 2010.

[MAI 16] MAILlEFERT M., SCRENCI N., «Ecologie industrielle et nouveaux modèles économiques. Quels enjeux de développement pour les territoires? », Colloque du RIODD 2016, Jul 2016, Saint-Étienne, France. hal-01350016, 2016

[MCD 11] MCDONOUgh W., BRAUngaRT M., Cradle to cradle : créer et recycler à l'infini, Éditions Alternatives, 2011.

[MEI 94] MEIJKAMP R., Service-Products, a sustainable approach ?, Eco-efficient Services Workshop, Wuppertal Institute, septembre, Wuppertal, Allemagne, 1994.

[MEI 96] MEIJKAmp R., Friday Washingday: Stimulating Technological Innovation by Developing New Product Use Routines. [Online] [1999-09-03], 1996. 
[MEI 00] MEIJKAMP R., Changing Consumer Behaviour Through Eco-Efficient Services : An Empirical Study of Car Sharing in The Netherlands, Thèse de doctorat, University of Technology, Delft, Pays-Bas, 308 p., 2000.

[MER 17] MERLIN-BROGNIART C, « Nature et dynamique de l'innovation des nouveaux modèles de croissance : le cas de l'écologie industrielle et de l'économie de la fonctionnalité », Innovations, n 54, p.65-95, 2017.

[MOA 06] MOATI PH., RANVIER M., SURY R., Bouquets pour répondre globalement aux besoins des clients. Éléments pour l'analyse économique d'une nouvelle forme d'organisation des marchés pour le régime de croissance postfordien, CREDOC, Cahiers de recherche, (230), novembre, 2006.

[MOA 08] MOATI Ph., Economie des bouquets, Éditions de l'aube, 2008.

[MON 02] MONT O., Clarifying the concept of product-service system, Journal of Cleaner Production, 10, n³, p. $237-$ 245, 2002.

[MON 04] MonT O., Product-service Systems: Panacea or Myth? - Doctoral dissertation, International Institute for Industrial Environmental Economics, Lund University, Sweden, 2004.

[MOO 96] MOORE J-F, The death of Competition. Leadership and Strategy in the Age of Business Ecosystems, Harper Business, New York, 1996.

[MOR 16] MORLAT C., « Modélisation dynamique des systèmes de coûts (MDSC) : un système de représentation des valeurs adapté à l'essor de l'économie de la fonctionnalité pour la transition écologique », Innovations, n²49,p.177201, 2016.

[PEA 76] PEARCE D.W., « The limits of costs benefit analysis as a guide to environmental policy », Kyklos, $\mathrm{n}^{\circ} 29$, p.97-112, 1976.

[POR 85] PORTER M., Competitive Advantage, New York: Free Press, 1985.

[REB 97] REBOUD L., "Introduction», dans La relation de service au cour de l'analyse économique, sous la direction de L. Reboud, coll. Logiques sociales, éd. L’Harmattan, 1997.

[RIF 00] RIFKIN J., The Age of Acess: The New Culture of Hypercapitalism Where All of Life Is a Paid For Experience, Putnam Publishing Group, 312p, 2000.

[RUD 00] RUDIN A., «Let's stop wasting energy on efficiency programs », Energy and Environment, 11, n5, p. 539$551,2000$.

[SCH 00] SCHIPPER L., «On the rebound : the interaction of energy efficiency, energy use and economic activity », Energy Policy, 28, n6-7, p. 351-353, 2000.

[SER 18] SERRA B., Perspectives durables et territoriales des économies de fonctionnalité légitimes. Une interprétation en termes conventionnalistes, Thèse dirigée par le Pr. BUCLET, Université Grenoble Alpes, 18 juin, 2018.

[SID 17] SIDOLI Y., L'usage en partage : Analyse comparative des modèles socio-économiques d'"économie de (la) fonctionnalité" et d'"économie collaborative, Thèse Sociologie. Université Côte d'Azur, 2017. <NNT : 2017AZUR2008>. <tel-01516611>, 2017.

[STA 94a] STAHEL W. R., «The Utilisation-Focused Service Economy: Resource Effiency and Product-Life Extension », dans Allenby B. R. (eds), The Greening of Industrial Ecosystems, National Academy Press, p. 91-100, 1994a.

[STA 94b] STAHEL W.R., Services and sustainability, Eco-efficient Services Workshop, Wuppertal Institute, Septembre, Wuppertal, Allemagne, 1994b.

[STA 97] STAHEL W. R., The Functional Economy: Cultural and Organizational Change from the Industrial Green Game: Implications for Environmental Design and Management, Washington DC, National Academy Press, 1997.

[STA 06] STAHEL W. R., The Performance Economy, Palgrave Macmillan, 2006.

[TAN 01] TANNERY F., «Le management stratégique des services : synthèse bibliographique et repérage des questions génériques », Finance Contrôle Stratégie, 4, n², p. 215-259, 2001.

[TIS 02] TISCHNER U., VerkuIJL M., TUKKER A., Product Service Systems: best practice document: SusProNet. 133 pp, 2002.

[TOF 02] TOFFEL M. W., Contracting for Servicizing, working paper, Harvard Business School, Boston, Massachusetts, May, 31p, 2002. 
[TUK 04] TUKKER A., « Eight types of Product-Service System: eight ways to sustainability? Experiences from Suspronet », Business Strategy and the Environment, 13, p. 246-260, 2004.

[TUK 06] TUKKER A., TISCHNER U. (eds), New business for old Europe: product-service development, competitiveness and sustainability, Greenleaf publishing, Sheffield, 2006.

[VAI 09] VAILEANU-PAUN I., Stratégies des entreprises et des territoires dans l'économie de la fonctionnalité. Le Cas EDF, Thèse de sciences économiques, Université Paris Diderot, 2009.

[VAI 10] VAILEANU-PAUN I., « Vers une territorialisation de la valeur des entreprises : les apports de l'économie de la fonctionnalité », Travail, Capital et Savoir dans la mondialisation, Mars 2010, Grenoble, France, 2010.

[VAN 88] VANDERMERWE S., RADA J.F., "Servitization of business: adding value by adding services », European Management Journal, 6, nº 4, p.314-324, 1988.

[VAN 07] VAN NIEL J., L'économie de fonctionnalité : définition et état de l'art, note de synthèse réalisée dans le cadre du groupe de travail "Economie de fonctionnalité" de la Commission Nationale du développement durable, mai, 2007.

[VAN 14] VAN NIEL J., «L'économie de fonctionnalité : principes, éléments de terminologie et proposition de typologie », Développement Durable et Territoires, 5, n¹, p.1-21, 2014.

[WHI 99] White A., Stoughton M., Feng L., The quiet transition, Agence de protection de l'environnement, Bureau des déchets solides, Tellus institute, Boston, Etats-Unis, 89p, 1999

[WIN 08] WINDRUM P., GARCíA-GOÑI M., « A neo-Schumpeterian model of health services innovation », Research Policy, 37, nº , p. 649-672, 2008.

[ZAC 07] ZACKLAD M., "L'économie de fonctionnalité encastrée dans la socio-économie des transactions coopératives : dynamique servicielle et fidélisation soutenable », Actes du Colloque de Cerisy 2006, L'Economie des Services pour un Développement Durable, Nouvelles Richesses, Nouvelles Solidarités, Heurgon, Landrieu (coord.), L'Harmattan, 2007. 